\title{
Thinking on the technology of Electronic Engineering Automation
}

\author{
Weijuan Dun ${ }^{1}$,Guangwei $\mathrm{Jia}^{2}$,Wei Yan ${ }^{3}$,Wei $\mathrm{Li}^{4}$,Xun Yang \\ ${ }^{1}$ Hebei University, Shijiazhuang, Hebei Province, 050100 \\ ${ }^{2}$ Chang'an University, Shijiazhuang, Hebei Province, 050100 \\ ${ }^{3}$ Beijing University of Technology, Shijiazhuang, Hebei Province, 050100 \\ ${ }^{4}$ China University of Geosciences, Shijiazhuang, Hebei Province, 050100 \\ ${ }^{5}$ Jilin University of Technology, Shijiazhuang, Hebei Province, 050100
}

Abstract: The main characteristic of computer automation technology is its computer function. Then it is combined with computer aided design, computer aided manufacturing, computer aided manufacturing and so on, so as to form today's electronic computer. It combines computer information technology, circuit theory, information analysis and so on, so as to enhance the performance and efficiency of the computer. This article mainly analyzes the present situation of electronic engineering automation technology, discusses the importance of electronic engineering automation technology, and its application field and the future development prospect, so as to initiate some related thinking and put forward some personal suggestions. For your reference.

Keywords: electronic engineering; automation; technical thinking

\section{Introduction}

The rapid development of social economy in our country has driven the rapid development of the automation technology of electronic engineering in our country. Electronic engineering automation technology is a new thing after the reform and opening up. It has rapidly grown up as an important part of modern society and core technology, Electronic information engineering automation technology, electronic computer automation technology, electrical engineering automation technology have been widely used. Electronic engineering automation technology has become an important object in the development of modern society. It brings great changes to people's life and production, and brings convenience from all aspects. It is hoped that the future development of electronic engineering automation technology will be better and better, and the application scope will be more and more wide.

\section{The necessity of application of automation technology in electronic engineering design}

\subsection{Automation technology is an effective way to promote the efficiency of electronic engineering design}

The biggest advantage of automation is to change the traditional form of manual control, and replace manual labor with mechanical equipment, which saves a lot of manpower and greatly improves the work efficiency. The designers of electronic engineering integrate the automation technology into the design, which can greatly improve the design level

Copyright (C) 2018 Weijuan Dun et al.

doi: $10.18686 /$ esta.v5i2.71

This is an open-access article distributed under the terms of the Creative Commons Attribution Unported License

(http://creativecommons.org/licenses/by-nc/4.0/), which permits unrestricted use, distribution, and reproduction in any medium, provided the original

work is properly cited. 
and efficiency of the current electronic engineering. At the same time, it can also promote the integration of mechanical and electrical implementation, and show the strict standards and diversified design of engineering design.

\subsection{Automation technology is an important method to improve the intelligent control level of electronic engineering design}

The main design of electronic engineering is to use electronic equipment and information system to complete a series of design, development, integration and application. Information is the key and foundation of the design of electronic engineering. The application of electronic engineering automation technology provides designers with many conveniences, so that they can accurately analyze and process information of high-volume, high-density and high frequency, and greatly enhance the efficiency of information collection, processing and integration. It lays a good foundation for the intelligence and automation of information ${ }^{[1]}$.

\section{Development status of Electronic Engineering Automation}

\subsection{Lack of experience in the application of Electronic Engineering Automation}

At the present stage, the rapid development of automation in China has also brought some problems. Because of the slow development of China's economy before, the electronic engineering technology in China started late. The research on it is not deep enough. There is not enough experience and development process is slow. The research methods and means of automation are relatively traditional. The research on automation in the past is mainly from the aspects of technology and equipment inspection, fault diagnosis and so on, so as to get some automation experience. This research method has many limitations, forming many problems, and hindering the further development of automation technology. In such a case, electronic products are difficult to repair once they are in trouble, so they can only be redesigned and manufactured by new ideas and plans, resulting in waste of resources. The development level of science and technology is relatively slow in China. Late research on automation, plus the lack of innovative talents, makes automation research stagnate. Automation technology cannot be widely applied. Such a dilemma is not only conducive to the development of automation, but also has a certain impact on the market. The stagnation of market development will also affect the development of electronic technology ${ }^{[2]}$.

\subsection{Widely used in all walks of life}

With the wide spread of knowledge in electronic engineering automation technology, people's knowledge is improving. All industries begin to understand the value of electronic engineering, so it has been widely applied and developed. The most widely used industry of electronic engineering automation technology should be power industry, manufacturing industry, construction industry, etc. ${ }^{[3]}$. The development of the automation technology of electronic engineering not only improves the work efficiency and quality, but also expands the economic benefit. It is also because of its value that it can be widely used in all walks of life, and at the same time, it has also been developed to a certain extent.

\section{Application of Electronic Engineering Automation}

\subsection{Application in the field of Auxiliary Office}

The amount of data in electronic information engineering is relatively large. If it is manual operation, it will bring some trouble to the enterprise, and it will take a long time. The application of automation technology has improved the situation to a certain extent, which not only improves the efficiency of employees, but also greatly increases the accuracy and timeliness of information processing. Therefore, enterprises should expand the application scope of automation technology, improve the efficiency of information operation, and ensure the accuracy of the data. A company or a factory will apply automated accounting machines, communication systems, control skills, and information processing in the accounting machine during the process of production and operation. In order to reduce the human error, the operator will use the accounting machine to deal with the information, thus reducing the workload and 
reducing the intensity of work. Generally, planners have no process of planning, but use automation to deepen the process of planning ${ }^{[4]}$. Thanks to the automation technology, it is convenient that do the unordered and cumbersome mechanical operation content with a bookkeeping machine. It not only saves time but also prevents the operation of the accounting machine.

\subsection{Application in computer integrated manufacturing}

Electronic information technology is a very tedious work, in the actual design process of electronic information, its design process and design link is not only more but also complex. And the process of product design is also required to cooperate with many aspects to achieve the smooth completion of the process. However, if the automation technology is used in the design and use of electronic products, the process of design can be simplified and the process is easier and more fluent. Designers can make use of automation to optimize and improve each link. Automation can also link every link to build a comprehensive system. The information generated in the process of design and production will convey the correct information at the right time, which greatly improves the overall design process and optimizes the final effect. At the same time, the design time will be greatly shortened, the work intensity and workload are reduced, and the quality of the product is improved. The application of automation brings great changes to the design and production of the enterprise ${ }^{[5]}$.

\subsection{Application in circuit analysis and computer control}

Circuit analysis and computer control are the core of electronic engineering design. The main aspects of application automation technology in circuit design and computer control are: Periodic non-sinusoidal current circuit contains the inductor single-phase, multi-phase circuit, dual-port network, three circuits, etc. The analysis of the feasibility of the circuit design can improve the scientificity and efficiency of the design circuit. Automation technology contains a lot of technology about computer control, so the application process of automation control is very similar to the application process of computer control, the application of automation technology to computer control can greatly improve the accuracy and efficiency of computer technology.

\section{Future development trend of Electronic Engineering Automation}

\subsection{Improve the stability of the automatic control system}

The automatic control system is often affected by various factors, resulting in the system operation is not very stable, which brings great economic loss for the enterprise. The use of intelligent technology can effectively deal with these problems, which will not affect the operation of the whole system. The operation process of the automation control system is more complex, it needs higher ability of staff to make the operation, and the work efficiency is not ideal; the combination of automation and intelligence can improve work efficiency, reduce workload, optimize operation process, reduce safety accidents, reduce the influence of various factors on the system, and improve the defense ability of the system. Make the system safe and stable operation ${ }^{[6]}$.

\subsection{High efficiency has become the development direction of Electronic Engineering}

The wide application of automation technology has made electronic engineering develop in the direction of more efficient. As an electronic engineering, the most important measure is speed and precision. They have been widely applied to the construction and control system of high-speed CPU, so the static and dynamic characteristics of electronic engineering have been effectively improved. The speed of the electronic engineering system and the efficiency of the electronic engineering have been greatly improved. Flexible development is the development trend of the intelligent technology of electronic engineering, which mainly includes the automation of the electrical automation control system and the data control system. The process of production should be strictly enforced and its maximum function should be brought into play. For electric automation system, we can choose some systems with strong operation and high coverage, which can be tailored. The main purpose is to meet the needs of different users. 


\subsection{Integration becomes the development direction of Electronic Engineering}

The wide application of automation technology in the field of electronic engineering makes integration into the direction of the development of electronic engineering automation. CPU chip can improve the speed of electrical engineering control software, so automation technology put forward higher requirements for it, and it will develop towards integration. High technology LED display technology provides more convenience for the carrying of electronic products, and product design is more and more humanized, and it can also increase the information capacity of large screen. The application of Internet technology and encapsulation technology in electronic engineering system is also the embodiment of intelligence. The streamlined product under this mode realizes the reduction of cost. In the system of electronic engineering automation, we classify the structure according to the modular system, so as to achieve more standardization and integration of the electronic engineering automation system. Moreover, the mode of increasing and decreasing the module is different from the construction method of building materials, thus forming a numerical control system at different levels.

\section{Thinking about Electronic Engineering Automation}

\subsection{Increase the strength of Education}

In order to cultivate more specialized electronic engineering automation personnel, it will be realized through education and teaching. At present, many universities in China have set up electrical automation courses, and they are also very interested in the input of teachers, so that they have trained a lot of electrical automation talents for our country. A large number of innovative and specialized electronic engineering automation personnel have been gushing, which has brought great impetus to the development of electronic engineering automation in China. The cultivation of high-end technology talents needs to make efforts in many ways. Therefore, the government, society and universities should increase training efforts, enrich and improve students' knowledge system, improve students' operation ability, open students' innovative thinking and cultivate students' creativity. Researchers need to study the automation of Electronic Engineering, the staff should enhance their ability of reform and innovation, increase their practical experience, expand the competitiveness of China's electronic engineering automation technology in the world, clarify the future direction of economic and social development, and create greater room for the development of electronic engineering automation.

\subsection{Increase innovation in the reform}

At present, China's research on electronic engineering automation, its innovation and reform ability is generally lagging behind other countries. This is mainly because of the interference of past experiences and patterns, and the lack of professional talents, which makes it difficult for people to jump out of the ordinary logic of thinking. Therefore, relevant educational scholars and researchers must reform and innovate their thinking, improve the ability of hands-on practice, broaden our current market of science and technology information and electronic engineering, and enhance the competitiveness of China's electronic engineering automation in the international market ${ }^{[7]}$.

\section{Conclusion}

The development of the economy has led to the development of science and technology. The progress of science and technology has promoted the speed of the development of electronic engineering automation in China. At present, automation technology has been widely applied to various electronic fields, but its problems still puzzle the researchers of automation, and the phenomenon of resource waste has not been improved. There are not many innovative personnel in automation. Therefore, in the future development of electronic engineering automation, we should constantly strengthen the training of talents, improve the level of technology operation, and continuously promote cooperation and exchanges between China and the international community, and achieve win-win situation. 


\section{References}

1. Shifang Dong. Several discussions on the new technology for the future development of power system automation [J]. Small and medium enterprise management and technology, 2011 (12).

2. Hongnian Zhu. Discussions on the application of computer network technology in electronic information engineering [J]. Information and communication, 2016, 01:167-168.

3. Gaoming Liu. Application of computer technology in power system automation [J].Journal of Beijing Electric Power College (Natural Science Edition), 2011 (08).

4. Wei Zhang. Analysis of the relationship between mechanical and electrical engineering and artificial intelligence [J]. Shandong Industrial Technology, 2016,04: 135.

5. Xuesong Han. Thinking on the technology of Electronic Engineering Automation [J]. Chinese and foreign entrepreneurs, 2016, (24): 121.

6. Jianguo Tan. Research on intelligent technology in automatic control of electronic engineering[J]. Electronic Engineering and Software Engineering, 2016, (10): 155-156.

7. Weiru Ren, Xiaoqin Ren. Discussion on the application of power automation technology in electric power engineering[J]. Power Technology, 2013 (9). 